\title{
Differential Calculi on Associative Algebras and Integrable Systems
}

\author{
Aristophanes Dimakis ${ }^{a}$ And Folkert MÜller-Hoissen ${ }^{b, c}$ \\ ${ }^{a}$ Dept. of Financial and Management Engineering, University of the Aegean, 82100 Chios, Greece \\ e-mail: dimakis@aegean.gr \\ ${ }^{b}$ Max Planck Institute for Dynamics and Self-Organization, 37077 Göttingen, Germany \\ ${ }^{c}$ Institute for Theoretical Physics, University of Göttingen, 37077 Göttingen, Germany \\ e-mail: folkert.mueller-hoissen@theorie.physik.uni-goettingen.de
}

\begin{abstract}
After an introduction to some aspects of bidifferential calculus on associative algebras, we focus on the notion of a "symmetry" of a generalized zero curvature equation and derive Bäcklund and (forward, backward and binary) Darboux transformations from it. We also recall a matrix version of the binary Darboux transformation and, inspired by the so-called Cauchy matrix approach, present an infinite system of equations solved by it. Finally, we sketch recent work on a deformation of the matrix binary Darboux transformation in bidifferential calculus, leading to a treatment of integrable equations with sources.
\end{abstract}

\section{Introduction}

Integrability of a partial differential and/or difference equation (PDDE), or more generally a system of such equations, is most frequently understood in the sense that the equations arise as the compatibility (or integrability) condition of a set of linear equations, a Lax system. For some integrable PDEs in two dimensions, the compatibility condition can be expressed as the condition of vanishing curvature of a connection, but this differential-geometric framework is too narrow. However, there is a generalization of the differential-geometric setting to a framework of "noncommutative geometry", preserving simple computational rules. A crucial point is the use of "generalized differential forms" instead of "generalized vector fields" or generalized derivations (see, e.g., 2, 32]) as the basic structure. Whereas, for example, discrete derivatives obey a modified derivation rule, on the level of forms one can preserve the simple graded derivation (Leibniz) rule for a generalized exterior derivative. Substantial results can then be derived by very simple and universal computations.

Let $\mathcal{A}$ be an associative algebra, over a field $\mathbb{K}$ of characteristic zero, and $(\boldsymbol{\Omega}, \delta)$ a differential calculus over $\mathcal{A}$. Here $\boldsymbol{\Omega}=\bigoplus_{k \geq 0} \boldsymbol{\Omega}^{k}$ is a graded algebra with $\boldsymbol{\Omega}^{0}=\mathcal{A}$ and $\mathcal{A}$-bimodules $\boldsymbol{\Omega}^{k}$, and $\delta$ is a derivation of degree one satisfying $\delta^{2}=0$. A connection on a left $\mathcal{A}$-module $\Gamma$ is a linear map $\nabla: \Gamma \rightarrow \boldsymbol{\Omega}^{1} \otimes_{\mathcal{A}} \Gamma$, such that $\nabla(f \gamma)=\delta f \otimes_{\mathcal{A}} \gamma+f \nabla \gamma$, for all $f \in \mathcal{A}$ and $\gamma \in \Gamma$. It extends to $\boldsymbol{\Omega} \otimes_{\mathcal{A}} \Gamma$, so that we can define the curvature as $\nabla^{2}$. The essence of integrability of some "condition" may then be expressed as

$$
\nabla^{2}=0 \quad \Longleftrightarrow \quad \text { condition . }
$$

For example, here "condition" could mean that a variable solves a certain PDDE. In this case we are looking for a differential calculus and a connection on a module, depending on the dependent variable of the PDDE, such that the zero curvature condition holds iff the variable satisfies the PDDE.

We will make the simplifying assumption that the module $\Gamma$ has a basis $b^{\mu}, \mu=1, \ldots, m$. Then, using the summation convention, we have $\gamma=\gamma_{\mu} b^{\mu}$ and

$$
\nabla \gamma=\left(\delta \gamma_{\mu}+\gamma_{\nu} \boldsymbol{A}_{\mu}^{\nu}\right) \otimes_{\mathcal{A}} b^{\nu}
$$




$$
\nabla^{2} \gamma=\gamma_{\mu} F_{\delta}[\boldsymbol{A}]^{\mu}{ }_{\nu} \otimes_{\mathcal{A}} b^{\nu}, \quad F_{\delta}[\boldsymbol{A}]^{\mu}{ }_{\nu}=\delta \boldsymbol{A}^{\mu}{ }_{\nu}-\boldsymbol{A}^{\mu}{ }_{\kappa} \boldsymbol{A}^{\kappa}{ }_{\nu},
$$

where $\boldsymbol{A}$ is the $m \times m$ matrix of elements of $\boldsymbol{\Omega}^{1}$ defined by $\nabla b^{\mu}=\boldsymbol{A}^{\mu}{ }_{\nu} \otimes_{\mathcal{A}} b^{\nu}$.

However, in most cases the above characterization of integrability is not strong enough. Rather, one needs a connection that depends on a ("spectral") parameter and the stronger condition that the curvature vanishes for all of its values. Bidifferential calculus [13, 20, is the special case where $\nabla$ is linear in such a parameter. In particular, one meets this situation in case of the (anti-) self-dual Yang-Mills equation [22,33. So let

$$
\delta=\overline{\mathrm{d}}+\lambda \mathrm{d}, \quad \boldsymbol{A}=A+\lambda B .
$$

The zero curvature condition is required to hold for all $\lambda$ and is then equivalent to

$$
F_{\overline{\mathrm{d}}}[A]=0=F_{\mathrm{d}}[B], \quad \mathrm{d} A+\overline{\mathrm{d}} B+A B+B A=0 .
$$

Since typically one of the two "gauge potentials" $A$ and $B$ can be transformed to zero by a gauge transformation, we set $B=01$ Then the zero curvature condition reduces to

$$
\mathrm{d} A=0, \quad F_{\overline{\mathrm{d}}}[A]=0 .
$$

The first equation can be solved by setting $A=\mathrm{d} \phi$, with $\phi \in \operatorname{Mat}(m, m, \mathcal{A})$, the algebra of $m \times m$ matrices over $\mathcal{A}$. Then the last equation takes the form

$$
\mathrm{d} \overline{\mathrm{d}} \phi+\mathrm{d} \phi \mathrm{d} \phi=0 \text {. }
$$

Alternatively, we can solve $F_{\overline{\mathrm{d}}}[A]=0$ by setting $A=(\overline{\mathrm{d}} g) g^{-1}$ with an invertible $g \in \operatorname{Mat}(m, m, \mathcal{A})$. Then $\mathrm{d} A=0$ results in

$$
\mathrm{d}\left[(\overline{\mathrm{d}} g) g^{-1}\right]=0 .
$$

Equations (1.2) and (1.3) are related by

$$
\overline{\mathrm{d}} g=(\mathrm{d} \phi) g,
$$

which has both, (1.2) and (1.3), as integrability conditions.

Remark 1.1. Another way to reduce the zero curvature condition to a single equation is by setting $A=(\overline{\mathrm{d}} g-(\mathrm{d} g) \Delta) g^{-1}$ with some $\Delta \in \mathcal{A}$, satisfying $\overline{\mathrm{d}} \Delta=(\mathrm{d} \Delta) \Delta$. Then (1.3) is generalized to $\mathrm{d}\left[(\overline{\mathrm{d}} g-(\mathrm{d} g) \Delta) g^{-1}\right]=0$.

The problem of finding a Lax system for a PDDE, or a system of PDDEs, is now replaced by the problem of finding a bidifferential calculus representation, such that the PDDE (system) is equivalent to either (1.2) or (1.3). These two equations can be regarded (cf. [20]) as generalizations or analogs of well-known potential forms of the famous (anti-) self-dual Yang-Mills equation on a flat four-dimensional space with Euclidean or split signature [22, 33. We should point out that there may be PDDEs possessing a Lax system, but no bidifferential calculus representation. At least quite a number of integrable PDDEs are indeed realizations of one of the above equations.

How to understand that an equation like (1.2) has so many integrable equations as realizations? Of course, in order to make contact with an integrable PDDE, the algebra $\mathcal{A}$ has to include an algebra of functions on some set. But we can extend it by operators, so that $\phi$ becomes operator-valued. If, nevertheless, (1.2) turns out to be equivalent to a PDDE, or a system of PDDEs, we are in business.

In the bidifferential calculus framework, we thus consider "universal" equations for objects in any associative algebra. Since there are many concrete choices of a bidifferential calculus, there are lots of different realizations, which inherit essential properties from the universal framework. (1.2) and (1.3) are important examples, but we will also show that they have

\footnotetext{
${ }^{1}$ Setting alternatively $A=0$ and keeping $B$, corresponds to an exchange of $\mathrm{d}$ and $\overline{\mathrm{d}}$ in the following equations.
} 
interesting extensions. It is important to stress that bidifferential calculus is a framework. It is not bound to the equations introduced above.

Although examples presented in this work are integrable PDDEs, we want to emphasize the general structure, which only requires a graded associative algebra and two anticommuting graded derivations of degree one acting on it with coboundary properties. There might well be applications to quite different areas of mathematics.

In Section 2 we define in a more precise way what we mean by a bidifferential calculus. Some important examples are recalled. Furthermore, it is shown that (1.1) is the integrability condition of a linear system. In this sense, (1.1) is integrable. Moreover, there is a simple construction of an infinite chain of conservation laws.

Section 3 introduces a class of symmetries of (1.1). We show that they lead to a universal Bäcklund transformation [37, 38] of (1.2), respectively (1.3), as well as to (forward, backward and binary) Darboux transformations (see [34 for concrete examples).

Perhaps the most efficient method to generate large classes of exact solutions of integrable systems is a matrix version of the binary Darboux transformation method. An abstract version in bidifferential calculus is recalled in Section 2, Section 5 presents new material, an infinite system of equations solved by the latter method.

In Section 6, we sketch recent work about a deformation of the binary Darboux transformation in bidifferential calculus, leading to integrable equations with sources [7, 36].

Finally, Section 7 contains some concluding remarks.

\section{Bidifferential calculus}

First we give a precise definition of bidifferential calculus.

Definition 2.1. A graded associative algebra is an associative algebra $\boldsymbol{\Omega}=\bigoplus_{r \geq 0} \boldsymbol{\Omega}^{r}$ over a field $\mathbb{K}$ of characteristic zero, where $\mathcal{A}:=\boldsymbol{\Omega}^{0}$ is an associative algebra over $\mathbb{K}$ and $\boldsymbol{\Omega}^{r}, r \geq 1$, are $\mathcal{A}$-bimodules such that $\boldsymbol{\Omega}^{r} \boldsymbol{\Omega}^{s} \subseteq \boldsymbol{\Omega}^{r+s}$. A bidifferential calculus is a unital graded associative algebra $\boldsymbol{\Omega}$, supplied with two $\mathbb{K}$-linear graded derivations d, $\overline{\mathrm{d}}: \boldsymbol{\Omega} \rightarrow \boldsymbol{\Omega}$ of degree one (hence $\left.\mathrm{d} \boldsymbol{\Omega}^{r} \subseteq \boldsymbol{\Omega}^{r+1}, \overline{\mathrm{d}} \boldsymbol{\Omega}^{r} \subseteq \boldsymbol{\Omega}^{r+1}\right)$, and such that

$$
\mathrm{d}^{2}=\overline{\mathrm{d}}^{2}=\mathrm{d} \overline{\mathrm{d}}+\overline{\mathrm{d}} \mathrm{d}=0 .
$$

The next subsection explains the integrability of the equations (1.2) and (1.3), respectively their predecessor (1.1), in bidifferential calculus. Another subsection recalls a construction of an infinite chain of conservation laws. The third subsection is devoted to integrable PDDEs as realizations of the equations (1.2) or (1.3) in bidifferential calculus. Further subsections deal with bidifferential calculi associated with compatible Lie algebra structures, and with a topic in differential geometry known as Frölicher-Nijenhuis theory.

\subsection{Linear systems and integrability}

Let $\Delta \in \operatorname{Mat}(n, n, \mathcal{A})$ and $\lambda$ be an $n \times n$ matrix of elements of $\Omega^{1}$, subject to the following equations,

$$
\overline{\mathrm{d}} \Delta+[\lambda, \Delta]=(\mathrm{d} \Delta) \Delta, \quad \overline{\mathrm{d}} \lambda+\lambda^{2}=(\mathrm{d} \lambda) \Delta .
$$

The linear system

$$
\overline{\mathrm{d}} \theta=A \theta+(\mathrm{d} \theta) \Delta+\theta \lambda,
$$

for $\theta \in \operatorname{Mat}(m, n, \mathcal{A})$, then has the integrability condition

$$
F_{\overline{\mathrm{d}}}[A] \theta-(\mathrm{d} A) \theta \Delta=0 .
$$

If $\Delta$ is such that this implies separate vanishing of both summands, (1.1) turns out to be a consistency condition of (2.3) . (1.1) is integrable in the sense that it arises as the integrability 
condition of a linear system. The equations (2.2) are in particular solved by $\lambda=0$ and a $\mathrm{d}-$ and $\overline{\mathrm{d}}$-constant $\Delta$. But in some cases other solutions of (2.2) are more important (see, e.g., [10,21]).

In the same way one finds that (1.1) is also the integrability condition of the "adjoint" linear system

$$
\overline{\mathrm{d}} \eta=-\eta A+\Gamma \mathrm{d} \eta+\kappa \eta,
$$

where $\eta \in \operatorname{Mat}(n, m, \mathcal{A}), \Gamma \in \operatorname{Mat}(n, n, \mathcal{A})$, and $\kappa$ is an $n \times n$ matrix of elements of $\boldsymbol{\Omega}^{1}$. They have to satisfy

$$
\overline{\mathrm{d}} \Gamma-[\kappa, \Gamma]=\Gamma \mathrm{d} \Gamma, \quad \overline{\mathrm{d}} \kappa-\kappa^{2}=\Gamma \mathrm{d} \kappa .
$$

(2.1) and the graded derivation property of $d$ and $\bar{d}$ are sufficient to obtain these results.

\subsection{A recursive construction of conservation laws}

Let $(\boldsymbol{\Omega}, \mathrm{d}, \overline{\mathrm{d}})$ be a bidifferential calculus. We call an element $J$ of $\boldsymbol{\Omega}^{1}$ a bi-closed 1 -form 2 if

$$
\mathrm{d} J=0 \quad \text { and } \quad \overline{\mathrm{d}} J=0 .
$$

Now let $J_{1}$ be a given bi-closed 1 -form. If $\mathrm{d} J_{1}=0$ implies the existence of an element $\chi_{1} \in \mathcal{A}$ such that

$$
J_{1}=\mathrm{d} \chi_{1}
$$

then it follows, by use of (2.1), that

$$
J_{2}=\overline{\mathrm{d}} \chi_{1}
$$

is also a bi-closed 1-form. This leads to a sequence of bi-closed 1-forms,

$$
J_{k+1}=\mathrm{d} \chi_{k+1}=\overline{\mathrm{d}} \chi_{k}, \quad k=1,2, \ldots,
$$

provided the closed d-forms appearing in the construction are d-exact (which is in particular true if the d-cohomology is trivial). Hence, if there is one bi-closed 1-form, there is typically an infinite number of bi-closed 1-forms.

How to find a bi-closed 1-form to start with? If $f \in \mathcal{A}$ satisfies $\overline{\mathrm{d}} \mathrm{d} f=0$, then $J=\overline{\mathrm{d}} f$ is a bi-closed 1-form of the bidifferential calculus, since $\overline{\mathrm{d}} J=\overline{\mathrm{d}}^{2} f=0$ and $\mathrm{d} J=\mathrm{d} \overline{\mathrm{d}} f=-\overline{\mathrm{d}} \mathrm{d} f=0$. In particular, any non-zero $f \in \mathcal{A}$ satisfying $\mathrm{d} f=0$ or $\overline{\mathrm{d}} f=0$ determines a bi-closed 1 -form, which can then be used to apply the above construction of a chain of bi-closed 1-forms.

Remark 2.2. If $J_{1}=\overline{\mathrm{d}} \chi_{0}$ and $\overline{\mathrm{d}} \mathrm{d} \chi_{0}=0$, the formal power series

$$
\chi=\sum_{k=0}^{\infty} \chi_{k} \mu^{k}
$$

with a parameter or an indeterminate $\mu$, satisfies the linear system

$$
\mathrm{d} \chi=\mu \overline{\mathrm{d}} \chi+\mathrm{d} \chi_{0} .
$$

The above construction becomes really useful by observing that the zero curvature condition (1.1) is equivalent to the bicomplex conditions

$$
\mathrm{d}^{2}=0, \quad \bar{D}^{2}=0, \quad \mathrm{~d} \bar{D}+\bar{D} \mathrm{~d}=0
$$

where we promoted d to the "generalized covariant exterior derivative"

$$
\bar{D}=\overline{\mathrm{d}}-A .
$$

\footnotetext{
${ }^{2}$ This generalizes the notion of a fundamental 1-form introduced in 31] to bidifferential calculus. In the spirit of 24, it may also be called a "conservation law". Also see Section 2.5.
} 
Now d and $\bar{D}$ are acting on $\operatorname{Mat}(m, m, \Omega)$, regarded as a left $\mathcal{A}$-module, and $\chi_{k} \in \operatorname{Mat}(m, m, \mathcal{A})$. If the bicomplex conditions are equivalent to some PDDE, for a particular choice of the bidifferential calculus, then the above construction, with $\overline{\mathrm{d}}$ replaced by $\bar{D}$, yields an infinite chain of conservation laws for this PDDE. This has been elaborated for many integrable equations [13, also see [14 16. As a special case, it includes the construction of non-local conservation laws for principal chiral models in [3], which has been formulated in terms of differential forms and generalized in [11,12].

\subsubsection{A modification}

Let $A=\mathrm{d} \phi$ and $\phi \in \operatorname{Mat}(m, m, \mathcal{A})$ be a solution of (1.2). Then $\mathrm{d}$ and $\bar{D}$ satisfy the bicomplex conditions.

We first note that (1.2) can be expressed as

$$
\mathrm{d}(\overline{\mathrm{d}} \phi+\phi \mathrm{d} \phi)=0 .
$$

If this implies that the 1 -form in the brackets is exact, there is $a_{1} \in \operatorname{Mat}(m, m, \mathcal{A})$ such that

$$
\mathrm{d} a_{1}=\overline{\mathrm{d}} \phi+\phi \mathrm{d} \phi .
$$

Acting with $\overline{\mathrm{d}}$ on this equation, using the latter to eliminate $\overline{\mathrm{d}} \phi$ and (1.2) to replace the term $\overline{\mathrm{d}} \mathrm{d} \phi$, leads to

$$
0=\overline{\mathrm{d}} \mathrm{d} a_{1}-\mathrm{d} a_{1} \mathrm{~d} \phi=-\mathrm{d}\left(\overline{\mathrm{d}} a_{1}+a_{1} \mathrm{~d} \phi\right) .
$$

We are thus led to

$$
\mathrm{d} a_{2}=\overline{\mathrm{d}} a_{1}+a_{1} \mathrm{~d} \phi .
$$

Continuing in this way and setting $a_{0}=\phi$, we obtain the recurrence relation

$$
\mathrm{d} a_{k+1}=\overline{\mathrm{d}} a_{k}+a_{k} \mathrm{~d} a_{0} \quad k=0,1, \ldots
$$

Furthermore, we have

$$
\overline{\mathrm{d}} \mathrm{d} a_{k}=\mathrm{d} a_{k} \mathrm{~d} \phi \quad k=0,1, \ldots
$$

Let now $\tilde{\chi}_{0} \in \operatorname{Mat}(m, m, \mathcal{A})$ be a solution of

$$
0=\mathrm{d} \bar{D} \tilde{\chi}_{0}=\mathrm{d}\left(\overline{\mathrm{d}} \tilde{\chi}_{0}-(\mathrm{d} \phi) \tilde{\chi}_{0}\right)=\mathrm{d}\left(\overline{\mathrm{d}} \tilde{\chi}_{0}+\phi \mathrm{d} \tilde{\chi}_{0}\right) .
$$

This means that 3

$$
\tilde{J}_{1}=\overline{\mathrm{d}} \tilde{\chi}_{0}+\phi \mathrm{d} \tilde{\chi}_{0}
$$

is d-closed. Assuming that it is d-exact, there is $\tilde{\chi}_{1} \in \operatorname{Mat}(m, m, \mathcal{A})$ such that

$$
\tilde{J}_{1}=\mathrm{d} \tilde{\chi}_{1},
$$

and it follows, by use of (2.6), that

$$
\tilde{J}_{2}=\overline{\mathrm{d}} \tilde{\chi}_{1}+a_{1} \mathrm{~d} \tilde{\chi}_{0}
$$

is d-closed. Assuming again d-exactness, we have

$$
\tilde{J}_{2}=\mathrm{d} \tilde{\chi}_{2} .
$$

\footnotetext{
${ }^{3}$ Here we depart from the previous scheme since $\tilde{J}_{1}=J_{1}+\mathrm{d}\left(\phi \tilde{\chi}_{0}\right)$, where $J_{1}=\bar{D} \tilde{\chi}_{0} . \tilde{J}_{1}$ is not annihilated by $\bar{D}$ if $\phi \neq 0$.
} 
Continuing in this way, by using (2.7) we obtain a chain of d-closed (and then d-exact) 1 -forms

$$
\tilde{J}_{k+1}:=\overline{\mathrm{d}} \tilde{\chi}_{k}+a_{k} \mathrm{~d} \tilde{\chi}_{0}=\mathrm{d} \tilde{\chi}_{k+1} \quad k=0,1, \ldots
$$

If $a_{k}=0, k=1,2, \ldots$, this reduces to the previous construction in Section 2.2 In the particular case of Frölicher-Nijenhuis theory, also see Section 2.5, this scheme reduces to that in [30] (also see 29]), where it is shown to appear even in the simple example of geodesic flow on $\mathbb{R}^{n}$.

According to the main scheme in Section 2.2 we can also construct a chain of elements $\chi_{k} \in \operatorname{Mat}(m, m, \mathcal{A})$ satisfying

$$
J_{k+1}:=\bar{D} \chi_{k}=\mathrm{d} \chi_{k+1} \quad k=0,1, \ldots,
$$

which only involves $a_{0}=\phi$, but not $a_{k}, k>0$. Setting $\tilde{\chi}_{0}=\chi_{0}$, we find

$$
\tilde{\chi}_{k}=\chi_{k}+\sum_{\substack{i+j=k-1 \\ i, j \geq 0}} a_{i} \chi_{j} \quad k=1,2, \ldots
$$

modulo adding a d-constant element of $\operatorname{Mat}(m, m, \mathcal{A})$.

Remark 2.3. In [29] an infinite chain of constants of motion of integrable hierarchies of hydrodynamic type were constructed using Frölicher-Nijenhuis theory (also see Section 2.5). This is an example of our construction recalled in Section 2.2. since the constants of motion are integrals of $\chi_{k}, k=0,1, \ldots$, which do not involve $a_{k}, k>0$. But the latter show up in the corresponding hierarchy of hydrodynamic type. We note that, in equation (2.24) of [29], the inverse of the relation (2.8) can be found, in different notation $\left(a_{i} \mapsto-a_{i}, k_{i}=\chi_{i}, h_{i}=\tilde{\chi}_{i}, i=0,1, \ldots\right)$.

All this motivates an exploration of the generalized scheme, presented above, in concrete examples. This will be left for future work.

\subsection{Integrable PDDEs obtained as realizations of equations in bidif- ferential calculus}

Given a unital associative algebra $\mathcal{A}$, a possible choice of $\Omega$ is

$$
\Omega=\mathcal{A} \otimes \bigwedge \mathbb{C}^{K},
$$

where $\bigwedge \mathbb{C}^{K}$ is the exterior algebra of the vector space $\mathbb{C}^{K}$. In this case it is sufficient to define $\mathrm{d}$ and $\overline{\mathrm{d}}$ on $\mathcal{A}$, since they extend in an evident way to $\Omega$, treating elements of $\Lambda \mathbb{C}^{K}$ as d- and $\overline{\mathrm{d}}$-constants. Let $\xi_{1}, \ldots, \xi_{K}$ be a basis of $\bigwedge^{1} \mathbb{C}^{K}$.

Prototype example: self-dual Yang-Mills. The (anti-) self-dual Yang-Mills equation on a flat four-dimensional space with Euclidean or split signature plays a prominent role as a fourdimensional integrable PDE system, from which many integrable PDEs can be derived via a reduction. It is also a prototype system in bidifferential calculus. Let $\mathcal{A}$ be the algebra of smooth complex functions of four (real or complex) variables $y, \bar{y}, z, \bar{z}$. Using (2.9) with $K=2$, we define

$$
\mathrm{d} f=-f_{y} \xi_{1}+f_{z} \xi_{2}, \quad \overline{\mathrm{d}} f=f_{\bar{z}} \xi_{1}+f_{\bar{y}} \xi_{2},
$$

on $\mathcal{A}$. Here a subscript indicates a partial derivative with respect to the corresponding independent variable. With the above calculus, it follows that (1.2) and (1.3) coincide with two well-known potential forms of the self-dual Yang-Mills equation (cf. 33]),

$$
\phi_{\bar{y} y}+\phi_{\bar{z} z}+\left[\phi_{y}, \phi_{z}\right]=0, \quad\left(g_{\bar{y}} g^{-1}\right)_{y}+\left(g_{\bar{z}} g^{-1}\right)_{z}=0 .
$$

Also see [20, for example.

Further examples. Many integrable equations can be obtained as a reduction of the selfdual Yang-Mills equation. This holds in particular for the chiral model equation governing 
stationary and axially symmetric solutions of Einstein's equation in electrovacuum, see [10,21] and references cited there. However, the Kadomtsev-Petviashvili (KP) equation apparently cannot be usefully obtained in this way. But it fits perfectly well into bidifferential calculus. Matrix versions of the most familiar integrable equations (including Korteweg-deVries $(\mathrm{KdV}) \sqrt[4]{4}$, Boussinesq, Sine-Gordon, Nonlinear Schrödinger equation and its integrable discretizations, KP, Davey-Stewartson, Toda and (generalized) Volterra lattices, two-dimensional Toda lattice, discrete KP and Hirota bilinear difference equation) have been treated in the bidifferential calculus framework, and corresponding infinite families of exact (soliton) solutions have been generated. These examples are all based on a graded algebra of the form (2.9). A comprehensive search for further examples still has to be carried out, with the aid of computer algebra.

\subsection{Compatible Lie algebra structures}

Let $\mathcal{V}$ be a vector space (over some field), supplied with a Lie algebra structure, which is given by a skew-symmetric map [, ] $: \mathcal{V} \otimes \mathcal{V} \rightarrow \mathcal{V}$, satisfying the Jacobi identity. Associated with it is the Chevalley-Eilenberg operator $\delta$, defined by

$$
\begin{aligned}
(\delta \mathfrak{f})\left(v_{1}, v_{2}, \ldots, v_{k+1}\right)= & \sum_{i=1}^{k+1}(-1)^{i+1}\left[v_{i}, \mathfrak{f}\left(v_{1}, \ldots, \hat{v}_{i}, \ldots, v_{k+1}\right)\right] \\
& +\sum_{i<j}(-1)^{i+j} \mathfrak{f}\left(\left[v_{i}, v_{j}\right], v_{1}, \ldots, \hat{v}_{i}, \ldots, \hat{v}_{j}, \ldots, v_{k+1}\right)
\end{aligned}
$$

acting on alternating multi-linear maps $\mathfrak{f}: \bigwedge^{k} \mathcal{V} \rightarrow \mathcal{V}$. Here a hat indicates an omission. Given two compatible Lie algebra structures (which means that any linear combination is also a Lie algebra structure), the corresponding Chevalley-Eilenberg operators $\delta_{1}$ and $\delta_{2}$ on $\boldsymbol{\Omega}=\mathcal{V} \oplus \bigoplus_{k=1}^{\infty} \operatorname{Hom}\left(\bigwedge^{k} \mathcal{V}, \mathcal{V}\right)$ constitute a bidifferential calculus. The case of two compatible Poisson structures on $\mathcal{V}=C^{\infty}(M)$, on a manifold $M$, makes contact with the framework of bi-Hamiltonian systems, also see the next subsection 5

\subsection{Frölicher-Nijenhuis theory}

A Nijenhuis tensor on a manifold $M$ is a tensor field $N$ of type $(1,1)$ (or a fiber preserving endomorphism of $T M$ ) on $M$, with vanishing Nijenhuis torsion, i.e.,

$$
T(N)(X, Y):=[N X, N Y]-N([N X, Y]+[X, N Y]-N[X, Y])=0,
$$

where $X, Y$ are vector fields on $M$, and [, ] denotes the usual commutator on the space of vector fields. In the context of integrable systems, we should mention the notion of a Poisson-Nijenhuis structure on $M$, which is given by a Poisson structure and a Nijenhuis tensor, satisfying a certain compatibility condition [27,31]. This structure has been shown to be an integrability feature of many integrable Hamiltonian systems.

Given a Nijenhuis tensor, a bidifferential calculus is obtained as follows. Let $\boldsymbol{\Omega}$ be the algebra of differential forms on $M$. Then the exterior derivative $\mathrm{d}$ and $\overline{\mathrm{d}}=\mathrm{d}_{N}:=i_{N} \mathrm{~d}$ satisfy (2.1). Here we look at $N$ as a vector-valued 1-form, and $i_{N}$ acts via contraction of a vector field and a 1-form. According to Frölicher-Nijenhuis theory [23, any derivation $\overline{\mathrm{d}}$ of degree one, which anti-commutes with $\mathrm{d}$ and satisfies $\overline{\mathrm{d}}^{2}=0$, is of the form $\mathrm{d}_{N}$.

In [30] a recursion operator on a symplectic manifold $(M, \omega)$ is defined to be a Nijenhuis tensor that is compatible with the symplectic 2 -form $\omega$. This means that $\omega(N X, Y)$ defines a 2-form and $\mathrm{d}_{N} \omega=0$. This reformulates a crucial structure of bi-Hamiltonian systems (a subclass of integrable systems), which serves to construct a so-called Lenard chain of conserved quantities in involution. We also refer to [1,4,6, 8, 9, 13, 28, 29, 40, for related work.

Using the (local d-) exactness of (d-) closed 1-forms, in [30] (also see [29]) the construction of a (generalized) Lenard chain is formulated concisely in terms of $\mathrm{d}$ and $\mathrm{d}_{N}$. This is a special case of

\footnotetext{
${ }^{4} \mathrm{~A}$ bidifferential calculus for $\mathrm{KdV}$ is recalled in Example 6.4 in Section 6 .

${ }^{5} \mathrm{~F}$ M-H had a very illuminating discussion about all this with Martin Bordemann in June 2000.
} 
the recursive construction of d-closed 1-forms in bidifferential calculus, presented in Section 2.2.1 In the present context, a bi-closed 1-form $J$ (cf. Section 2.2) is defined by $\mathrm{d} J=0=\mathrm{d}_{N} J$ and called a fundamental 1-form [31]. In [24] it has also been called a conservation law for a type $(1,1)$ tensor field $N$.

Remark 2.4. Via $\left(N^{*} \alpha\right)(X):=\alpha(N X), N$ determines an endomorphism $N^{*}$ of the space of 1-forms. The (generalized) Hodge star operator used in [11,12 is a linear map on the space of 1 -forms and thus plays the role of $N^{*}$.

\section{Symmetries in bidifferential calculus}

Let us consider the system (1.1). A symmetry of it should be any transformation of $A$ that leaves the system invariant, after implementing the condition that $A$ is a solution. The first of equations (1.1) is obviously invariant under

$$
A \mapsto A^{\prime}=A+\mathrm{d} \sigma,
$$

with a 0 -form (element of $\boldsymbol{\Omega}^{0}$ ) $\sigma$. The symmetry condition obtained from the second of equations (1.1) is then

$$
\mathrm{d}(\overline{\mathrm{d}} \sigma-[A, \sigma]+\sigma \mathrm{d} \sigma)=0,
$$

where we used that $A$ shall be a solution of (1.1). If we assume that the d-closed 1-form in the brackets is d-exact, there is a "potential" $\rho$ such that

$$
\overline{\mathrm{d}} \sigma-[A, \sigma]+\sigma \mathrm{d} \sigma=\mathrm{d} \rho .
$$

In the following we show that Bäcklund transformations and Darboux transformations are special classes of such symmetries. Bäcklund transformations arise via elimination of $\sigma$ from the symmetry conditions. In case of Darboux transformations, a solution $\sigma$ is constructed by use of the linear or adjoint linear system.

\subsection{Bäcklund transformation}

We show how Bäcklund transformations emerge from the above class of symmetries. If we set $\rho=0$ and use $\mathrm{d} \sigma=A^{\prime}-A$, we obtain

$$
\overline{\mathrm{d}} \sigma=A \sigma-\sigma A^{\prime} .
$$

There are now the following two cases (also see [17, 20]).

1. Writing $A=\mathrm{d} \phi$ and $A^{\prime}=\mathrm{d} \phi^{\prime}$, the equation $\mathrm{d} \sigma=A^{\prime}-A$ is solved by

$$
\sigma=\phi^{\prime}-\phi+C,
$$

where $\mathrm{d} C=0$. The remaining symmetry condition then reads

$$
\overline{\mathrm{d}}\left(\phi^{\prime}-\phi+C\right)=\mathrm{d} \phi\left(\phi^{\prime}-\phi+C\right)-\left(\phi^{\prime}-\phi+C\right) \mathrm{d} \phi^{\prime}, \quad \mathrm{d} C=0 .
$$

This is a relation between two solutions of (1.2) and thus constitutes a Bäcklund transformation for the equation (1.2).

2. Alternatively, using $A=(\overline{\mathrm{d}} g) g^{-1}$ and $A^{\prime}=\left(\overline{\mathrm{d}} g^{\prime}\right) g^{\prime-1}$, we are led to

$$
\sigma=g K g^{\prime-1}, \quad \overline{\mathrm{d}} K=0,
$$

and thus

$$
\left(\overline{\mathrm{d}} g^{\prime}\right) g^{{ }^{-1}}-(\overline{\mathrm{d}} g) g^{-1}=\mathrm{d}\left(g K g^{\prime-1}\right), \quad \overline{\mathrm{d}} K=0 .
$$

This is a Bäcklund transformation for the equation (1.3).

In 17] we derived and recovered Bäcklund transformations from the above general expressions for quite a number of integrable equations. 


\subsubsection{Permutability}

Let $A_{i}, A_{k}$ be two solutions of (1.1), related by a Bäcklund transformation, hence

$$
\mathrm{d} \sigma_{i, k}=A_{k}-A_{i}, \quad \overline{\mathrm{d}} \sigma_{i, k}=A_{i} \sigma_{i, k}-\sigma_{i, k} A_{k} .
$$

This can be expressed as a refactorization condition,

$$
\left(\overline{\mathrm{d}}-A_{i}-\lambda^{-1} \mathrm{~d}\right) \mathcal{B}(\lambda)_{i, k}=\mathcal{B}(\lambda)_{i, k}\left(\overline{\mathrm{d}}-A_{k}-\lambda^{-1} \mathrm{~d}\right) \quad \forall \lambda,
$$

acting from the left on $\operatorname{Mat}(m, m, \Omega)$. Here we set

$$
\mathcal{B}(\lambda)_{i, k}=1+\lambda \sigma_{i, k} .
$$

Suppose there are two different chains of Bäcklund transformations,

$$
A_{i} \stackrel{\mathcal{B}(\lambda)_{i, k}}{\longmapsto} A_{k} \stackrel{\mathcal{B}(\lambda)_{k, j}}{\longmapsto} A_{j}, \quad A_{i} \stackrel{\mathcal{B}(\lambda)_{i, l}}{\longmapsto} A_{l} \stackrel{\mathcal{B}(\lambda)_{l, j}}{\longmapsto} A_{j},
$$

starting with the same solution and ending with the same solution. Then the refactorization equation implies

$$
\mathcal{B}(\lambda)_{i, k} \mathcal{B}(\lambda)_{k, j}=\mathcal{B}(\lambda)_{i, l} \mathcal{B}(\lambda)_{l, j} \quad \forall \lambda,
$$

which requires the permutability conditions

$$
\sigma_{i, k}+\sigma_{k, j}=\sigma_{i, l}+\sigma_{l, j}, \quad \sigma_{i, k} \sigma_{k, j}=\sigma_{i, l} \sigma_{l, j} .
$$

Referring to the two cases above, this becomes

$$
\begin{aligned}
\left(\phi_{k}-\phi_{i}+C_{i, k}\right)\left(\phi_{j}-\phi_{k}+C_{k, j}\right) & =\left(\phi_{l}-\phi_{i}+C_{i, l}\right)\left(\phi_{j}-\phi_{l}+C_{l, j}\right), \\
C_{i, k}+C_{k, j} & =C_{i, l}+C_{l, j},
\end{aligned}
$$

respectively

$$
\begin{aligned}
g_{i} K_{i, k} g_{k}^{-1}+g_{k} K_{k, j} g_{j}^{-1} & =g_{i} K_{i, l} g_{l}^{-1}+g_{l} K_{l, j} g_{j}^{-1} \\
K_{i, k} K_{k, j} & =K_{i, l} K_{l, j} .
\end{aligned}
$$

For concrete choices of bidifferential calculi associated with integrable PDDEs, corresponding "permutability theorems" 37, 38 arise from these equations. If two solutions are obtained by (elementary) Bäcklund transformations (involving different parameters) applied to a given solution, then a fourth solution is determined in a purely algebraic way. See [17] for many examples, where permutability theorems are recovered via the bidifferential calculus approach.

\subsection{Forward Darboux transformation}

For given solutions $\Delta_{\theta}, \lambda_{\theta}$ of (2.2), with $n=m$, and a solution $A_{0}$ of (1.1), let $\theta \in \operatorname{Mat}(m, m, \mathcal{A})$ be an invertible solution of the linear system

$$
\overline{\mathrm{d}} \theta=A_{0} \theta+(\mathrm{d} \theta) \Delta_{\theta}+\theta \lambda_{\theta},
$$

which is (2.3) with $n=m$ and $A$ replaced by $A_{0}$. Here solutions of (2.2) carry a subscript $\theta$ just to remind us of the fact that these are the solutions of (2.2) that appear as coefficients in the linear equation for $\theta$. This convention will be used further on in the present section, but not beyond it.

Setting

$$
\sigma_{[+1]}=\theta \Delta_{\theta} \theta^{-1}
$$

it follows that

$$
A_{[+1]}=A_{0}+\mathrm{d} \sigma_{[+1]}
$$


also satisfies (1.1). Let $\psi$ be a solution of the linear system

$$
\overline{\mathrm{d}} \psi=A_{0} \psi+(\mathrm{d} \psi) \Delta_{\psi}+\psi \lambda_{\psi},
$$

where $\Delta_{\psi}, \lambda_{\psi}$ satisfy (2.2). Then

$$
\psi_{[+1]}=\sigma_{[+1]} \psi-\psi \Delta_{\psi}
$$

satisfies

$$
\overline{\mathrm{d}} \psi_{[+1]}=A_{[+1]} \psi_{[+1]}+\left(\mathrm{d} \psi_{[+1]}\right) \Delta_{\psi}+\psi_{[+1]} \lambda_{\psi} .
$$

\subsection{Backward Darboux transformation}

For given solutions $\Gamma_{\eta}, \kappa_{\eta}$ of (2.5) with $n=m$, and a solution $A_{0}$ of (1.1), let $\eta \in \operatorname{Mat}(m, m, \mathcal{A})$ be an invertible solution of the adjoint linear system

$$
\overline{\mathrm{d}} \eta=-\eta A_{0}+\Gamma_{\eta} \mathrm{d} \eta+\kappa_{\eta} \eta,
$$

which is (2.4) with $n=m$, and $A_{0}$ instead of $A$. Setting

$$
\sigma_{[-1]}=-\eta^{-1} \Gamma_{\eta} \eta,
$$

it follows that

$$
A_{[-1]}=A_{0}+\mathrm{d} \sigma_{[-1]}
$$

also satisfies (1.1). Let $\chi$ be a solution of the linear system

$$
\overline{\mathrm{d}} \chi=-\chi A_{0}+\Gamma_{\chi} \mathrm{d} \chi+\kappa_{\chi} \chi,
$$

where $\Gamma_{\chi}, \kappa_{\chi}$ satisfy (2.5). Then

$$
\chi_{[-1]}=\Gamma_{\chi} \chi+\chi \sigma_{[-1]}
$$

satisfies

$$
\overline{\mathrm{d}} \chi_{[-1]}=-\chi_{[-1]} A_{[-1]}+\Gamma_{\chi} \mathrm{d} \chi_{[-1]}+\kappa_{\chi} \chi_{[-1]} .
$$

\subsection{Binary Darboux transformation}

The following essentially generalizes the binary Darboux transformation method as formulated, e.g., in [34, to bidifferential calculus. In order to combine forward and backward Darboux transformations, we have to look for a transformation of $\chi$ under the forward Darboux transformation and a transformation of $\psi$ under the backward Darboux transformation. Let $\Omega(\chi, \psi)$ satisfy the consistent linear equations

$$
\begin{aligned}
\Gamma_{\chi} \Omega(\chi, \psi)-\Omega(\chi, \psi) \Delta_{\psi}= & \chi \psi, \\
\overline{\mathrm{d}} \Omega(\chi, \psi)= & (\mathrm{d} \Omega(\chi, \psi)) \Delta_{\psi}-\left(\mathrm{d} \Gamma_{\chi}\right) \Omega(\chi, \psi) \\
& +(\mathrm{d} \chi) \psi+\kappa_{\chi} \Omega(\chi, \psi)+\Omega(\chi, \psi) \lambda_{\psi} .
\end{aligned}
$$

Then

$$
\psi_{[-1]}:=\eta^{-1} \Omega(\eta, \psi), \quad \chi_{[+1]}:=\Omega(\chi, \theta) \theta^{-1},
$$

indeed satisfy the linear equations

$$
\begin{aligned}
& \overline{\mathrm{d}} \psi_{[-1]}=A_{[-1]} \psi_{[-1]}+\left(\mathrm{d} \psi_{[-1]}\right) \Delta_{\psi}+\psi_{[-1]} \lambda_{\psi}, \\
& \overline{\mathrm{d}} \chi_{[+1]}=-\chi_{[+1]} A_{[+1]}+\Gamma_{\chi} \mathrm{d} \chi_{[+1]}+\kappa_{\chi} \chi_{[+1]} .
\end{aligned}
$$


Inserting

$$
\theta_{[-1]}=\eta^{-1} \Omega(\eta, \theta), \quad \eta_{[+1]}=\Omega(\eta, \theta) \theta^{-1},
$$

in the expressions for $\sigma_{[ \pm 1]}$, yields

$$
\begin{aligned}
\sigma_{[+1,-1]} & :=-\eta_{[+1]}^{-1} \Gamma_{\eta} \eta_{[+1]}=-\theta \Omega(\eta, \theta)^{-1} \Gamma_{\eta} \Omega(\eta, \theta) \theta^{-1}, \\
\sigma_{[-1,+1]} & :=\theta_{[-1]} \Delta_{\theta} \theta_{[-1]}^{-1}=\eta^{-1} \Omega(\eta, \theta) \Delta_{\theta} \Omega(\eta, \theta)^{-1} \eta,
\end{aligned}
$$

and we obtain

$$
\begin{aligned}
A_{[+1,-1]} & :=A_{[+1]}+\mathrm{d} \sigma_{[+1,-1]}=A_{0}-\mathrm{d}\left(\theta \Omega(\eta, \theta)^{-1} \eta\right) \\
& \left.=A_{[-1]}+\mathrm{d} \sigma_{[-1,+1]}\right)=: A_{[-1,+1]} .
\end{aligned}
$$

Furthermore,

$$
\begin{aligned}
& \chi_{[+1,-1]}=\Gamma_{\chi} \chi_{[+1]}+\chi_{[+1]} \sigma_{[+1,-1]}=\chi-\Omega(\chi, \theta) \Omega(\eta, \theta)^{-1} \eta, \\
& \psi_{[-1,+1]}=\sigma_{[-1,+1]} \psi_{[-1]}-\psi_{[-1]} \Delta_{\theta}=\psi-\theta \Omega(\eta, \theta)^{-1} \Omega(\eta, \psi),
\end{aligned}
$$

satisfy the linear equations

$$
\begin{aligned}
& \overline{\mathrm{d}} \chi_{[+1,-1]}=-\chi_{[+1,-1]} A_{[+1,-1]}+\Gamma_{\chi} \mathrm{d} \chi_{[+1,-1]}+\kappa_{\chi} \chi_{[+1,-1]}, \\
& \overline{\mathrm{d}} \psi_{[-1,+1]}=A_{[-1,+1]} \psi_{[-1,+1]}+\left(\mathrm{d} \psi_{[-1,+1]}\right) \Delta_{\psi}+\psi_{[-1,+1]} \lambda_{\psi} .
\end{aligned}
$$

The transformation

$$
\left(A_{0}, \psi, \chi\right) \longmapsto\left(A_{[+1,-1]}, \psi_{[-1,+1]}, \chi_{[+1,-1]}\right)
$$

is the binary Darboux transformation.

1. Using $A=\mathrm{d} \phi$, we see that

$$
\phi_{[+1,-1]}=\phi_{[-1,+1]}=\phi_{0}-\theta \Omega(\eta, \theta)^{-1} \eta
$$

solves (1.2).

2. Using instead $A=(\overline{\mathrm{d}} g) g^{-1}$, we conclude that

$$
g_{[-1,+1]}=g_{[+1,-1]}=\left(1-\theta \Omega(\eta, \theta)^{-1} \Gamma_{\eta}^{-1} \eta\right) g_{0}
$$

solves (1.3). We note that

$$
g_{[-1,+1]}^{-1}=g_{0}^{-1}\left(1+\theta \Delta_{\theta}^{-1} \Omega(\eta, \theta)^{-1} \eta\right) .
$$

Remark 3.1. Setting

$$
q=\eta_{[+1]}^{-1}, \quad r=\theta_{[-1]}^{-1},
$$

we find that they satisfy the linear system

$$
\begin{aligned}
& \overline{\mathrm{d}} q=A_{[+1,-1]} q+(\mathrm{d} q) \Gamma_{\eta}+q\left(\mathrm{~d} \Gamma_{\eta}-\kappa_{\eta}\right), \\
& \overline{\mathrm{d}} r=-r A_{[-1,+1]}+\Delta_{\theta} \mathrm{d} r+\left(\mathrm{d} \Delta_{\theta}-\lambda_{\theta}\right) r,
\end{aligned}
$$

from which we read off that $\Delta_{q}=\Gamma_{\eta}, \lambda_{q}=\mathrm{d} \Gamma_{\eta}-\kappa_{\eta}, \Gamma_{r}=\Delta_{\theta}$ and $\kappa_{r}=\mathrm{d} \Delta_{\theta}-\lambda_{\theta}$. Furthermore, we have

$$
\Delta_{\theta} \Omega(r, q)-\Omega(r, q) \Gamma_{\eta}=r q,
$$

and

$$
\overline{\mathrm{d}} \Omega(r, q)=(\mathrm{d} \Omega(r, q)) \Gamma_{\eta}-\left(\mathrm{d} \Delta_{\theta}\right) \Omega(r, q)+(\mathrm{d} r) q
$$




$$
\begin{aligned}
& +\left(\mathrm{d} \Delta_{\theta}-\lambda_{\theta}\right) \Omega(r, q)+\Omega(r, q)\left(\mathrm{d} \Gamma_{\eta}-\kappa_{\eta}\right) \\
= & \left(\mathrm{d} \Omega(r, q) \Gamma_{\eta}\right)+(\mathrm{d} r) q-\Omega(r, q) \kappa_{\eta}-\lambda_{\theta} \Omega(r, q) .
\end{aligned}
$$

Inserting our expressions for $q$ and $r$, we can conclude that these equations are solved by setting

$$
\Omega(r, q)=-\Omega(\eta, \theta)^{-1}
$$

Moreover, one can show that

$$
\Omega\left(r, \psi_{[-1,+1]}\right)=\Omega(\eta, \theta)^{-1} \Omega(\eta, \psi), \quad \Omega\left(\chi_{[+1,-1]}, q\right)=\Omega(\chi, \theta) \Omega(\eta, \theta)^{-1} .
$$

Using $q$ and $r$ in the binary Darboux transformation, we find

$$
\begin{aligned}
& A_{[+1,-1]}-\mathrm{d}\left(q \Omega(r, q)^{-1} r\right)=A_{0}, \\
& \psi_{[-1,+1]}-q \Omega(r, q)^{-1} \Omega\left(r, \psi_{[-1,+1]}\right)=\psi, \\
& \chi_{[-1,+1]}-\Omega\left(\chi_{[-1,+1]}, q\right) \Omega(r, q)^{-1}=\chi .
\end{aligned}
$$

Hence this leads back to what we started with.

From the above Darboux transformations one recovers known Darboux transformations 34] for many integrable systems via corresponding choices of the bidifferential calculus.

\section{A matrix version of the binary Darboux transformation}

Now we drop the restriction $n=m$, imposed in the preceding section. Let us recall that (1.2) is integrable in the sense that it arises as the integrability condition of the linear system

$$
\overline{\mathrm{d}} \theta=(\mathrm{d} \phi) \theta+(\mathrm{d} \theta) \Delta+\theta \lambda .
$$

This is (2.3) with $A=\mathrm{d} \phi$. Of course, $\Delta$ and $\lambda$ have to satisfy (2.2).

(1.2) is also the integrability condition of the "adjoint" linear system

$$
\overline{\mathrm{d}} \eta=-\eta \mathrm{d} \phi+\Gamma \mathrm{d} \eta+\kappa \eta,
$$

where $\Gamma$ and $\kappa$ have to solve (2.5).

Let $\Omega$ be a solution of the linear equations

$$
\begin{aligned}
& \Gamma \Omega-\Omega \Delta=\eta \theta, \\
& \overline{\mathrm{d}} \Omega=(\mathrm{d} \Omega) \Delta-(\mathrm{d} \Gamma) \Omega+\kappa \Omega+\Omega \lambda+(\mathrm{d} \eta) \theta .
\end{aligned}
$$

The equation obtained by acting with $\overline{\mathrm{d}}$ on (4.3) is identically satisfied as a consequence of (4.1), (4.2), (4.3), and the equation that results from (4.3) by acting with $\mathrm{d}$ on it. Correspondingly, also the equation that results from acting with $\overline{\mathrm{d}}$ on (4.4) is identically satisfied as a consequence of the preceding equations. If $\phi_{0}$ is a given solution of (1.2), it follows [20,21] that

$$
\phi=\phi_{0}-\theta \Omega^{-1} \eta
$$

is a new solution of (1.2), and

$$
q=\theta \Omega^{-1}, \quad r=\Omega^{-1} \eta
$$

satisfy

$$
\overline{\mathrm{d}} q=(\mathrm{d} \phi) q+\mathrm{d}(q \Gamma)-q \kappa, \quad \overline{\mathrm{d}} r=-r \mathrm{~d} \phi+\mathrm{d}(\Delta r)-\lambda r .
$$

There is an analogous solution-generating result for equation (1.3), see [7] and references cited there. 


\section{An infinite system of equations in bidifferential calculus with solutions generated by the binary Darboux transfor- mation}

Are there perhaps other equations in bidifferential calculus that can also be solved via the binary

Darboux transformation? Let

$$
\Phi_{i, j}=\theta \Delta^{i} \Omega^{-1} \Gamma^{j} \eta \quad i, j \in \mathbb{Z} .
$$

A corresponding generating function is

$$
\Phi(s, t)=\theta(1-s \Delta)^{-1} \Omega^{-1}(1-t \Gamma)^{-1} \eta,
$$

which has the following formal power series expansions,

$$
\begin{array}{ll}
\Phi(s, t)=\sum_{i, j=0}^{\infty} \Phi_{i, j} s^{i} t^{j} \quad \text { around }(s, t)=(0,0), \\
\Phi(s, t)=-\sum_{i=1, j=0}^{\infty} \Phi_{-i, j} s^{-i} t^{j} & \text { around }(s, t)=(\infty, 0), \\
\Phi(s, t)=-\sum_{i=0, j=1}^{\infty} \Phi_{i,-j} s^{i} t^{-j} & \text { around }(s, t)=(0, \infty), \\
\Phi(s, t)=\sum_{i, j=1}^{\infty} \Phi_{-i,-j} s^{-i} t^{-j} & \text { around }(s, t)=(\infty, \infty) .
\end{array}
$$

Proposition 5.1. The following recurrence relation holds,

$$
\overline{\mathrm{d}} \Phi_{i, j}=\mathrm{d} \Phi_{i, j+1}-\left(\mathrm{d} \Phi_{i, 0}\right) \Phi_{0, j}=\mathrm{d} \Phi_{i+1, j}+\Phi_{i, 0} \mathrm{~d} \Phi_{0, j} \quad i, j \in \mathbb{Z} .
$$

Proof. By a direct computation we obtain

$$
\overline{\mathrm{d}} \Phi(s, t)+[\mathrm{d} \Phi(s, 0)] \Phi(0, t)=t^{-1} \mathrm{~d}[\Phi(s, t)-\Phi(s, 0)] .
$$

Expansion leads to the asserted sequence of equations.

Acting with d on (5.1) implies

$$
\mathrm{d} \overline{\mathrm{d}} \Phi_{i, j}-\mathrm{d} \Phi_{i, 0} \mathrm{~d} \Phi_{0, j}=0 \quad i, j \in \mathbb{Z} .
$$

In particular, setting $i=j=0$, we see that $\phi=-\Phi_{0,0}$ solves (1.2), which is the binary Darboux transformation result for vanishing seed solution.

Example 5.2. Choosing $i=0$ and $j=-1$ in (5.2), leads to

$$
\overline{\mathrm{d}}\left(1-\Phi_{0,-1}\right)+\left(\mathrm{d} \Phi_{0,0}\right)\left(1-\Phi_{0,-1}\right)=0 .
$$

As a consequence, if $g:=\left(1-\Phi_{0,-1}\right) g_{0}$, where $g_{0}$ is d- and $\overline{\mathrm{d}}$-constant, is invertible, then it solves (1.3). In a similar way, choosing $i=-1$ and $j=0$, we find that $h:=h_{0}\left(1+\Phi_{-1,0}\right)$, with a d- and $\overline{\mathrm{d}}$-constant $h_{0}$, solves $\mathrm{d}\left(h^{-1} \overline{\mathrm{d}} h\right)=0$, provided its inverse exists.

\section{Lemma 5.3.}

$$
\Phi_{i,-1} \Phi_{-1, j}=\Phi_{i-1, j}-\Phi_{i, j-1} \quad i, j \in \mathbb{Z} .
$$

Proof. A direct computation shows that

$$
\Phi(s, 0) \Phi(0, t)=t^{-1}[\Phi(s, t)-\Phi(s, 0)]-s^{-1}[\Phi(s, t)-\Phi(0, t)] .
$$


Expansion yields

$$
\Phi_{i+1, j}-\Phi_{i, j+1}+\Phi_{i, 0} \Phi_{0, j}=0 \quad i, j \in \mathbb{Z} .
$$

By repeated application of this relation, we find

$$
\begin{aligned}
\Phi_{i,-1} \Phi_{-1, j} & =\left(\Phi_{i-1,0}-\Phi_{i-1,0} \Phi_{0,-1}\right)\left(\Phi_{0, j-1}+\Phi_{-1,0} \Phi_{0, j-1}\right) \\
& =\Phi_{i-1,0} \Phi_{0, j-1}+\Phi_{i-1,0}\left(\Phi_{-1,0}-\Phi_{0,-1}-\Phi_{0,-1} \Phi_{-1,0}\right) \Phi_{0, j-1} \\
& =\Phi_{i-1, j}-\Phi_{i, j-1}+\Phi_{i-1,0}\left(\Phi_{-1,0} \Phi_{0,-1}-\Phi_{0,-1} \Phi_{-1,0}\right) \Phi_{0, j-1} \\
& =\Phi_{i-1, j}-\Phi_{i, j-1},
\end{aligned}
$$

using $\Phi_{-1,0} \Phi_{0,-1}=\Phi_{0,-1} \Phi_{-1,0}$, which is a consequence of (4.3).

\section{Proposition 5.4.}

$$
\overline{\mathrm{d}} \mathrm{d} \Phi_{i, j}=-\left(\overline{\mathrm{d}} \Phi_{i,-1}\right) \overline{\mathrm{d}} \Phi_{-1, j} \quad i, j \in \mathbb{Z} .
$$

Proof. A special case of (5.1) is

$$
\left(\overline{\mathrm{d}} \Phi_{i,-1}\right) \Phi_{-1, j}=\left(\mathrm{d} \Phi_{i, 0}\right)\left(\Phi_{-1, j}-\Phi_{0,-1} \Phi_{-1, j}\right) .
$$

Using the lemma, this becomes

$$
\left(\overline{\mathrm{d}} \Phi_{i,-1}\right) \Phi_{-1, j}=\left(\mathrm{d} \Phi_{i, 0}\right) \Phi_{0, j-1} .
$$

From (5.1) we now obtain

$$
\mathrm{d} \Phi_{i, j}=\overline{\mathrm{d}} \Phi_{i, j-1}+\left(\mathrm{d} \Phi_{i, 0}\right) \Phi_{0, j-1}=\overline{\mathrm{d}} \Phi_{i, j-1}+\left(\overline{\mathrm{d}} \Phi_{i,-1}\right) \Phi_{-1, j},
$$

which implies our assertion by acting with $\overline{\mathrm{d}}$ on it.

Choosing $i=j=-1$ in the preceding proposition, we obtain the next result.

Corollary 5.5. $\Phi_{-1,-1}$ solves

$$
\overline{\mathrm{d}} \mathrm{d} \varphi+\overline{\mathrm{d}} \varphi \overline{\mathrm{d}} \varphi=0,
$$

which is (1.2) with $\mathrm{d}$ and $\overline{\mathrm{d}}$ exchanged.

Remark 5.6. The construction of equations in this section, and corresponding solutions, is analogous to the "Cauchy matrix approach", see [26,41,42].

\section{Deformation of binary Darboux transformations and in- tegrable systems with sources}

Let us replace $\Omega$ by $\Omega-\omega$ in the equations of Section 4 , i.e.,

$$
\begin{aligned}
& \Gamma(\Omega-\omega)-(\Omega-\omega) \Delta=\eta \theta, \\
& \overline{\mathrm{d}}(\Omega-\omega)=\mathrm{d}(\Omega-\omega) \Delta-(\mathrm{d} \Gamma)(\Omega-\omega)+\kappa(\Omega-\omega)+(\Omega-\omega) \lambda+(\mathrm{d} \eta) \theta .
\end{aligned}
$$

Hence

$$
\begin{aligned}
& \Gamma \Omega-\Omega \Delta=\eta \theta+c, \\
& \overline{\mathrm{d}} \Omega=(\mathrm{d} \Omega) \Delta-(\mathrm{d} \Gamma) \Omega+\kappa \Omega+\Omega \lambda+(\mathrm{d} \eta) \theta+\gamma,
\end{aligned}
$$

where

$$
c:=\Gamma \omega-\omega \Delta, \quad \gamma:=\overline{\mathrm{d}} \omega-(\mathrm{d} \omega) \Delta+(\mathrm{d} \Gamma) \omega-\kappa \omega-\omega \lambda .
$$

We note that they satisfy

$$
\begin{aligned}
& \overline{\mathrm{d}} \gamma=(\mathrm{d} \gamma) \Delta-(\mathrm{d} \Gamma) \gamma+\kappa \gamma-\gamma \lambda-(\mathrm{d} \kappa) c \\
& \overline{\mathrm{d}} c=(\mathrm{d} c) \Delta+\kappa c+c \lambda+\Gamma \gamma-\gamma \Delta
\end{aligned}
$$

By straightforward computations, one proves the following. 
Theorem 6.1 (7). Let $\Delta, \Gamma, \kappa, \lambda$ satisfy (2.2) and (2.5). Let $\phi_{0}$ be a solution of (1.2) and $\theta$, $\eta, \Omega$ satisfy the linear equations [4.1), (4.2) and (6.1), respectively. Then

$$
\phi=\phi_{0}-\theta \Omega^{-1} \eta, \quad q=\theta \Omega^{-1}, \quad r=\Omega^{-1} \eta,
$$

are solutions of

$$
\mathrm{d} \overline{\mathrm{d}} \phi+\mathrm{d} \phi \mathrm{d} \phi=\mathrm{d}(q \gamma r-q \mathrm{~d}(c r))
$$

and

$$
\begin{aligned}
& \overline{\mathrm{d}} q=(\mathrm{d} \phi) q+\mathrm{d}(q \Gamma)-q \kappa-q \gamma \Omega^{-1}-(\mathrm{d} q) c \Omega^{-1} \\
& \overline{\mathrm{d}} r=-r \mathrm{~d} \phi+\mathrm{d}(\Delta r)-\lambda r-\Omega^{-1} \gamma r+\Omega^{-1} \mathrm{~d}(c r) .
\end{aligned}
$$

Remark 6.2. The above equations form a consistent system in the sense that any equation derived from it by acting with $\overline{\mathrm{d}}$ on any of its members yields an equation that is satisfied as a consequence of the system.

Remark 6.3. Using the last two equations in (6.4), we eliminate $\theta$ and $\eta$ in (6.1) to obtain

$$
\begin{aligned}
& \Delta \hat{\Omega}-\hat{\Omega} \Gamma=r q+\hat{\Omega} c \hat{\Omega}, \\
& \overline{\mathrm{d}} \hat{\Omega}=\mathrm{d}(\hat{\Omega} \Gamma)-\hat{\Omega} \kappa-\lambda \hat{\Omega}+(\mathrm{d} r) q+((\mathrm{d} \hat{\Omega}) c+\hat{\Omega} \gamma) \hat{\Omega}
\end{aligned}
$$

where $\hat{\Omega}=-\Omega^{-1}$. (6.6) then reads

$$
\begin{aligned}
& \overline{\mathrm{d} q}=(\mathrm{d} \phi) q+\mathrm{d}(q \Gamma)-q \kappa+q \gamma \hat{\Omega}+(\mathrm{d} q) c \hat{\Omega}, \\
& \overline{\mathrm{d}} r=-r \mathrm{~d} \phi+\mathrm{d}(\Delta r)-\lambda r+\hat{\Omega} \gamma r-\hat{\Omega} \mathrm{d}(c r) .
\end{aligned}
$$

Together with (6.5), this constitutes a system of equations for $\phi, q, r$ and $\hat{\Omega}$, for which we now have a solution-generating method at hand.

Example 6.4. Let $\mathcal{A}_{0}$ be the space of smooth complex functions on $\mathbb{R}^{2}$. We extend it to $\mathcal{A}=\mathcal{A}_{0}[\partial]$, where $\partial$ is the partial differentiation operator with respect to the coordinate $x$. On $\mathcal{A}$ we define

$$
\mathrm{d} f=[\partial, f] \xi_{1}+\frac{1}{2}\left[\partial^{2}, f\right] \xi_{2}, \quad \overline{\mathrm{d}} f=-\frac{1}{2}\left[\partial^{2}, f\right] \xi_{1}+\frac{1}{3}\left[\partial_{t}-\partial^{3}, f\right] \xi_{2}
$$

(cf. [20]). The maps d and $\overline{\mathrm{d}}$ extend to linear maps on $\boldsymbol{\Omega}=\mathcal{A} \otimes \wedge \mathbb{C}^{2}$. Choosing

$$
\Delta=\Gamma=-\partial, \quad \kappa=\frac{1}{2} Q^{2}\left(\xi_{1}+\partial \xi_{2}\right), \quad \lambda=-\frac{1}{2} P^{2}\left(\xi_{1}+\partial \xi_{2}\right),
$$

with constant matrices $P, Q,(2.2)$ and (2.5) are satisfied. Requiring $\omega_{x}=0$ means $c=0$. Furthermore, we are led to set

$$
\gamma=\gamma_{1} \xi_{1}+\left(\gamma_{2}+\gamma_{1} \partial\right) \xi_{2}, \quad \gamma_{1}=-\frac{1}{2}\left(Q^{2} \omega-\omega P^{2}\right), \quad \gamma_{2}=\frac{1}{3} \omega_{t} .
$$

1. Let $\omega$ be constant, so that $\gamma_{2}=0$. Then, in terms of $u=2 \phi_{x}$, (6.5) and (6.6) lead to6

$$
\begin{aligned}
4 u_{t}-u_{x x x}-3\left(u^{2}\right)_{x} & =\left(q_{x} r-q r_{x}\right)_{x}, \\
q_{t}-q_{x x x}-\frac{1}{4} q r q & =\frac{3}{4}\left(u_{x} q+2 u q_{x}\right), \\
r_{t}-r_{x x x}+\frac{1}{4} r q r & =\frac{3}{4}\left(r u_{x}+2 r_{x} u\right),
\end{aligned}
$$

after a redefinition of $q$. Here we disregarded those equations resulting from (6.6) that involve $\gamma_{1}$. The above system is the second member of the Yajima-Oikawa hierarchy (also see the appendix

\footnotetext{
${ }^{6}$ There is a wrong factor in front of the cubic nonlinearities in (4.5) of 7 .
} 
of 7 ) 7 The first equation is a KdV equation with "sources".

2. Setting $\gamma_{1}=0$, we are led to

$$
4 u_{t}-u_{x x x}-3\left(u^{2}\right)_{x}=8\left(q \omega_{t} r\right)_{x}, \quad q_{x x}=q Q^{2}-u q, \quad r_{x x}=P^{2} r-r u .
$$

where $\omega_{t}$ can be absorbed by a redefinition of $q$ or $r$. Here we disregarded equations resulting from (6.6) that involve $\gamma_{2}$. This example of a "system with self-consistent sources" appeared in 35 .

In both cases, Theorem 6.1 generates exact solutions. Soliton solutions are obtained starting with vanishing seed solution. Many further examples can be found in [7].

\section{Conclusions and further remarks}

Bidifferential calculus is a drastic abstraction of structures that are relevant in the theory of (completely) integrable PDDEs. This structure may well find applications far away from the latter. This suggests to look for associative algebras that admit a bidifferential calculus. So far, there are only few examples beyond differential geometry (Frölicher-Nijenhuis theory) and examples with a graded algebra of the form (2.9), which underlies most of our work on integrable PDDEs. For example, in [39] a bidifferential calculus has been found for the quantum group $U_{q}(\operatorname{sl}(2))$, with $q$ a third root of unity. There is a vast literature by now on differential calculi on some classes of associative algebras. For any given $(\boldsymbol{\Omega}, \mathrm{d})$, the question is then whether there exists another graded derivation $\overline{\mathrm{d}}$ on $\boldsymbol{\Omega}$ that extends it to a bidifferential caclulus. For instance, this problem could be addressed for (bicovariant) differential calculi on Hopf algebras (quantum groups). Of particular interest are also deformations of algebras that underly classical integrable systems, leading to a kind of "quantization" of the latter. What we are looking for, in particular, is a noncommutative version of Frölicher-Nijenhuis theory.

Even concerning classical integrable systems, bidifferential calculus has not been explored systematically, so far. Given a bidifferential calculus, equations like (1.2) or (1.3) are not necessarily equivalent to a non-trivial PDDE (or a system of PDDEs). How to express the dependent variable in a suitable way in terms of the non-commuting elements of the algebra $\mathcal{A}$ ? So far this is still too much based on trial and error, and more systematics would be desirable.

We mention the following in order to build a bridge to other contributions to these proceedings, dealing with deformed derivations. Let the space of 1-forms $\boldsymbol{\Omega}^{1}$ admit a finite left and also right $\mathcal{A}$-module basis $\theta^{s}, s=1, \ldots, S$. Then there are maps $\Theta_{s^{\prime}}^{s}: \mathcal{A} \rightarrow \mathcal{A}$ such that

$$
f \theta^{s}=\sum_{s^{\prime}=1}^{S} \theta^{s^{\prime}} \Theta_{s^{\prime}}^{s}(f),
$$

for all $f \in \mathcal{A}$. Introducing generalized (left and right) partial derivatives via

$$
\mathrm{d} f=\sum_{s=1}^{S}\left(\partial_{s}^{(L)} f\right) \theta^{s}=\sum_{s=1}^{S} \theta^{s}\left(\partial_{s}^{(R)} f\right),
$$

the Leibniz rule yields

$$
\begin{aligned}
\sum_{i=1}^{J} \theta^{s} \partial_{s}^{(R)}(f h) & =\mathrm{d}(f h)=(\mathrm{d} f) h+f \mathrm{~d} h \\
& =\sum_{s=1}^{S} \theta^{s}\left(\partial_{s}^{(R)} f\right) h+f \sum_{s^{\prime}=1}^{S} \theta^{s^{\prime}} \partial_{s^{\prime}}^{(R)} h \\
& \left.=\sum_{s=1}^{S} \theta^{s}\left[\partial_{s}^{(R)} f\right) h+\sum_{s^{\prime}=1}^{S} \Theta_{s}^{s^{\prime}}(f) \partial_{s^{\prime}}^{(R)} h\right]
\end{aligned}
$$

\footnotetext{
${ }^{7}$ We are grateful to Dmitry Demskoi and Maxim Pavlov for informing us about this.
} 
Hence the right partial derivatives satisfy the twisted Leibniz rule

$$
\partial_{s}^{(R)}(f h)=\left(\partial_{s}^{(R)} f\right) h+\sum_{s^{\prime}=1}^{S} \Theta_{s}^{s^{\prime}}(f) \partial_{s^{\prime}}^{(R)} h .
$$

If $\Theta$ is diagonal, then the maps $\Theta_{s}^{s}$ are automorphisms and $\partial_{s}^{(R)}$ is a generalized derivation [2,32, (called $\sigma$-derivation in [25, for example). Also see [18,19] for corresponding examples. There is a similar formula for the left derivatives, of course, and corresponding generalized partial derivatives can also be associated with $\overline{\mathrm{d}}$. If the aforementioned conditions are met, equations considered in this work can be expressed in terms of these generalized partial derivatives.

\section{References}

[1] A. Arsie, A., Lorenzoni, P.: F-manifolds with eventual identities, bidifferential calculus and twisted Lenard-Magri chains. Int. Math. Res. Not. 2013, no. 17, 3931-3976 (2013).

[2] Boutet de Monvel, A., Marchenko, V.: Generalization of the Darboux transform. Mat. Fiz. Anal. Geom. 1, 479-504 (1994).

[3] Brezin, E., Itzykson, C., Zinn-Justin, J., Zuber, J.-B.: Remarks about the existence of nonlocal charges in two-dimensional models. Phys. Lett. B 82, 442-444 (1979).

[4] Camacaro, J.R., Moreno, C.: Conserved currents in Kähler manifolds. In: Ocampo, H., Pariguán, E., Paycha, S. (eds.) Geometric and Topological Methods for Quantum Field Theory, pp. 368-380. Cambridge University Press, Cambridge (2010).

[5] Chavchanidze, G.: Non-Noether symmetries and their influence on phase space geometry. J. Geom. Phys. 48, 190-202 (2003).

[6] __ : Non-Noether symmetries in Hamiltonian dynamical systems. Mem. Differential Equations Math. Phys. 36, 81-134 (2005).

[7] Chvartatskyi, O., Dimakis, A., Müller-Hoissen, F.: Self-consistent sources for integrable equations via deformations of binary Darboux transformations. Lett. Math. Phys. 106, 1139-1179 (2016).

[8] Crampin, M., Sarlet, W.: Bi-quasi-Hamiltonian systems. J. Math. Phys. 43, 2505-2517 (2002).

[9] Crampin, M., Sarlet, W., Thompson, G.: Bi-differential calculi and bi-Hamiltonian systems, J. Phys. A: Math. Gen. 33, L177-L180 (2000).

[10] Dimakis, A., Kanning, N., Müller-Hoissen, F.: The non-autonomous chiral model and the Ernst equation of General Relativity in the bidifferential calculus framework. SIGMA 7, 118 (2011).

[11] Dimakis, A., Müller-Hoissen, F.: Integrable discretizations of chiral models via deformation of the differential calculus. J. Phys. A: Math. Gen. 29, 5007-5018 (1996).

[12] _ : Noncommutative geometry and integrable models. Lett. Math. Phys. 39, 69-79 (1997).

[13] __ Bi-differential calculi and integrable models. J. Phys. A: Math. Gen. 33, 957-974 (2000).

[14] _ : Bicomplexes and finite Toda lattices. In: Doebner, H.-D., Dobrev, V.K., Hennig, J.-D., Lücke, W. (eds.) Quantum Theory and Symmetries, pp. 545-549. World Scientific, Singapore (2000).

[15] _ : Bicomplexes and integrable models. J. Phys. A: Math. Gen. 33, 6579-6591 (2000).

[16] _ The Korteweg-de-Vries equation on a noncommutative space-time. Phys. Lett. A 278, 139-145 (2000).

[17] _ _ Bicomplexes and Bäcklund transformations. J. Phys. A: Math. Gen. 34, 9163-9194 (2001). 
[18] _ : Automorphisms of associative algebras and noncommutative geometry. J. Phys. A: Math. Gen. 37, 2307-2330 (2004).

[19] _ : Differential calculi on quantum spaces determined by automorphisms. Czech J. Phys. 54, 1235-1241 (2004).

[20] _ : Bidifferential graded algebras and integrable systems. Discr. Cont. Dyn. Systems Suppl. 2009, 208-219 (2009).

[21] _ _ Binary Darboux transformations in bidifferential calculus and integrable reductions of vacuum Einstein equations. SIGMA 9, 009 (2013).

[22] Dunajski, M.: Solitons, Instantons and Twistors. Oxford Graduate Texts in Mathematics, vol. 19. Oxford University Press, Oxford (2009).

[23] Frölicher, A., Nijenhuis, A.: Theory of vector-valued differential forms. Part I. Derivations in the graded ring of differential forms. Proc. Koninkl. Ned. Acad. Wetensch. Ser. A 59, $338-359$ (1956).

[24] Grifone, J., Mehdi, M.: Existence of conservation laws and characterization of recursion operators for completely integrable systems. Transactions AMS 349, 4609-4633 (1997).

[25] Hartwig, J.T., Larsson, D., Silvestrov, S.D.: Deformations of Lie algebras using $\sigma$ derivations. J. Algebra 295, 314-361 (2006).

[26] Hietarinta, J., Joshi, N., Nijhoff, F.: Discrete systems and integrability. Cambridge University Press, Cambridge (2016).

[27] Kosmann-Schwarzbach, Y., Magri, F.: Poisson-Nijenhuis structures. Ann. Inst. Henri Poincaé section A 53, 35-81 (1990).

[28] Lorenzoni, P.: Flat bidifferential ideals and semi-Hamiltonian PDEs. J. Phys. A: Math. Gen. 39, 13701-13716 (2006).

[29] Lorenzoni, P., Magri, F.: A cohomological construction of integrable hierarchies of hydrodynamic type. Int. Math. Res. Not. 34, 2087-2100 (2005).

[30] Magri, F.: Lenard chains for classical integrable systems. Theor. Math. Phys. 137, 1716$1722(2003)$.

[31] Magri, F., Morosi, C.: A geometrical characterization of integrable Hamiltonian systems through the theory of Poisson-Nijenhuis manifolds. Quaderno S 19, 1-176 (1988).

[32] Marchenko, V.A.: Nonlinear Equations and Operator Algebras. Mathematics and Its Applications. Reidel, Dordrecht (1988).

[33] Mason, L.J., Woodhouse, N.M.J.: Integrability, Self-Duality, and Twistor Theory. Clarendon Press, Oxford (1996).

[34] Matveev, V.B., Salle, M.A.: Darboux Transformations and Solitons. Springer Series in Nonlinear Dynamics. Springer, Berlin (1991).

[35] Mel'nikov, V.K.: Integration method of the Korteweg-de Vries equation with a selfconsistent source. Phys. Lett. A 133, 493-496 (1989).

[36] Müller-Hoissen, F., Chvartatskyi, O., Toda, K.: Generalized Volterra lattices: Binary Darboux transformations and self-consistent sources. J. Geom. Phys. 113, 226-238 (2017).

[37] Rogers, C., Schief, W.K.: Bäcklund and Darboux Transformations. Cambridge Texts in Applied Mathematics. Cambridge University Press, Cambridge (2002).

[38] Rogers, C., Shadwick, W.F.: Bäcklund Transformations and Their Applications. Academic Press, New York (1982).

[39] Sitarz, A.: Finite Hopf algebras and bidifferential structures. Lett. Math. Phys. 51, 205-209 (2000).

[40] Tondo, G.: Generalized Lenard chains and separation of variables. Quaderni Matematici dell'Università di Trieste 573, 1-27 (2006). 
[41] Xu, D.-D., Zhang, D.-J., Zhao, S.-L.: The Sylvester equation and integrable equations: I. The Korteweg-de Vries system and sine-Gordon equation. J. Nonl. Math. Phys. 21, 382-406 (2014).

[42] Zhang, D.-J., Zhao, S.-L.: Solutions of the ABS lattice equations via generalized Cauchy matrix approach. Stud. Appl. Math. 131, 72-103 (2013). 